\title{
Delaying meiotic resumption during transportation of bovine cumulus-oocyte complexes: effects on development, apoptosis and caspases activity of in vitro-produced embryos
}

\author{
Priscila Chediek Dall'Acqua ${ }^{2,3}$, Beatriz Caetano da Silva Leão ${ }^{2,3}$, \\ Nathália Alves de Souza Rocha-Frigoni ${ }^{2,3}$, Fernanda Patrícia Gottardi ${ }^{4}$ and Gisele Zoccal Mingoti ${ }^{1,3}$ \\ UNESP, School of Veterinary Medicine, Laboratory of Reproductive Physiology, Araçatuba; UNESP, School of Agricultural \\ and Veterinarian Sciences, Graduate Program in Veterinary Medicine, Jaboticabal; and UFPI, Bom Jesus, Brazil
}

Date submitted: 09.03.2017. Date revised: 11.08.2017. Date accepted: 21.10.2017

\section{Summary}

This study examined the effects of meiosis inhibition during bovine oocyte transportation on developmental competence and quality of produced embryos. The transportation medium was supplemented with: $100 \mu \mathrm{M}$ butyrolactone I (BL), $500 \mu \mathrm{M}$ IBMX $+100 \mu \mathrm{M}$ forskolin (mSPOM), $100 \mu \mathrm{M}$ milrinone (MR) or follicular fluid (bFF), and was carried out in a portable incubator for $6 \mathrm{~h}$. Next, oocytes were in vitro matured (IVM) for $18 \mathrm{~h}$, without the meiotic inhibitors, with the exception of mSPOM group, in which was added $20 \mu \mathrm{M}$ cilostamide. The three control groups were IVM with $10 \%$ fetal calf serum (FCS) (Control Lab FCS) or $0.6 \%$ bovine serum albumin (BSA) (Control Lab BSA) in a $\mathrm{CO}_{2}$ in air incubator or in the portable incubator with $0.6 \%$ BSA (Control Transp BSA). Higher cleavage rates $(P<0.05)$ were obtained in the Control Lab FCS group $(84.5 \pm 5.3 \%)$ compared with the other groups $(59.6 \pm 3.4 \%$ to $70.9 \pm 2.3 \%)$. Embryonic development was higher $(P<0.05)$ in the Control Lab FCS group $(39.8 \pm 4.7 \%)$ than in the Control Transp BSA $(22.7 \pm 3.4 \%)$ and MR $(21.6 \pm 2.3 \%)$ groups. However, they were similar $(P>0.05)$ to the other groups $(23.6 \pm 3.3 \%$ to $28.8 \pm 2.7 \%)$. The total number of blastomeres was higher $(P<0.05)$ in the Control Lab FCS group $(85.2 \pm 5.6)$ than in Control Lab BSA $(53.6 \pm 2.9)$, Control Transp BSA (55.5 \pm 4.4$), \mathrm{BL}(58.2 \pm 3.0), \operatorname{mSPOM}(57.9 \pm 4.9)$ and MR (59.2 \pm 3.9$)$, but all these treatments did not differ $(P>0.05)$ from bFF $(67.7 \pm 4.2)$. No differences $(P>0.05)$ were found in apoptosis by the activity of caspases (139.0 \pm 3.2 to $152.4 \pm 6.5$, expressed in fluorescence intensity) as well as the percentage of TUNEL-positive cells $(12.3 \pm 2.0 \%$ to $15.7 \pm 1.7 \%)$. In conclusion, the transportation of oocytes over $6 \mathrm{~h}$ with $\mathrm{BL}, \mathrm{mSPOM}$ or bFF enabled the acquisition of developmental competence at similar rates to the Control Lab FCS group.

Keywords: Developmental competence, In vitro maturation, Meiotic block, Oocyte, Transport

\footnotetext{
${ }^{1}$ All correspondence to: G.Z. Mingoti. Universidade Estadual Paulista (UNESP), School of Veterinary Medicine, Department of Animal Health, Araçatuba 16050-680, São Paulo, Brazil. Tel: +55 183636 1375. Fax: +55 183636 1352. E-mail: gmingoti@fmva.unesp.br

${ }^{2}$ Universidade Estadual Paulista (UNESP), School of Veterinary Medicine, Laboratory of Reproductive Physiology, Araçatuba, SP 16050-680, Brazil.

${ }^{3}$ Universidade Estadual Paulista (UNESP), School of Agricultural and Veterinarian Sciences, Graduate Program in Veterinary Medicine, Jaboticabal, SP 16050-680, Brazil. ${ }^{4}$ Federal University of Piauí (UFPI), Bom Jesus, PI, Brazil.
}

\section{Introduction}

In vitro embryo production (IVP) on a commercial scale has increased significantly in recent years. As a consequence of the improvement of this biotechnology, the global production of bovine embryos by ovum pick-up (OPU) and in vitro fertilization (IVF) exceeded the halfmillion mark in 2013 (Perry, 2015). The commercial IVP of bovine requires the collection of cumulus-oocyte complexes (COCs) by OPU performed on-farm and their transportation to an IVP laboratory. COCs are usually transported in a bicarbonate-based maturation medium and placed in a $5 \% \mathrm{CO}_{2}$ in air gas-equilibrated 
incubator or in pre-equilibrated, gassed and sealed vials to maintain the physiological pH (BarcelóFimbres et al., 2015). However, in herds maintained distant from the laboratory, transportation of COCs may take several hours and decrease COC viability and competence, representing a challenge for commercial settings. Nevertheless, limited information exists on oocyte collection, transportation, and maturation with the objective to improve embryo production using assisted reproduction technologies.

In growing and dominant follicles, oocytes remain arrested at the diplotene stage of the meiotic prophase. In vivo, resumption of meiosis is initiated by a preovulatory luteinizing hormone ( $\mathrm{LH})$ surge and only occurs in fully grown meiotically competent oocytes, i.e., in those oocytes that have acquired the ability to resume and complete meiosis. The LH surge leads to a disruption in gap junctions that connect the oocyte with its surrounding cumulus cells, resulting in the disappearance of the highly coordinated bidirectional communication between these cells. During the period between the LH surge and ovulation, the oocyte undergoes a series of marked changes not only in its nucleus but also in its cytoplasm, the process being known as oocyte maturation (van den Hurk \& Zhao, 2005).

Thus, oocyte maturation in vivo is precisely controlled by maternal signals from cumulus cells that coordinate major oocyte meiotic and cytoplasmic events, and components that are required for complete developmental competence of the oocyte (Gilchrist \& Thompson, 2007; Albuz et al., 2010; Gilchrist, 2011). However, in vitro maturation (IVM) is likely to occur in the absence of these critical maternal signals, as precocious oocyte meiotic resumption irrevocably occurs when meiotically competent oocytes were aspirated from the inhibitory environment of the ovarian antral follicles by OPU and cultured in a suitable culture medium (Gilchrist \& Thompson, 2007), in a process called spontaneous oocyte maturation. During spontaneous resumption of meiosis, the chromatin condenses thus preventing RNA synthesis and probably curtailing the accumulation of several molecules essential for early embryo development (BilodeauGoeseels, 2012). Then, it was suggested that inhibition or delay of spontaneous nuclear maturation in vitro would allow more time for some of the oocytes to accumulate more of the molecules that are important for early development, which could therefore improve the efficiency of embryo IVP (reviewed by BilodeauGoeseels, 2012; Hashimoto et al., 2002).

Meiotic block has been induced by several strategies including the use of biological or pharmacological inhibitors. Bovine follicular fluid (bFF) has been used as a natural substance for blocking the meiosis (Sirard et al., 1998) due to an inhibitory effect on germinal vesicle breakdown, thus leading to a delay on the nuclear maturation (Ducolomb et al., 2013). Among pharmacological inhibitors, several agents that experimentally elevate oocyte cyclic adenosine monophosphate (cAMP) transiently inhibit the spontaneous maturation of bovine oocytes isolated from their follicles in vitro. These include non-specific (3isobutyl-1-methylxanthine - IBMX) or oocyte-specific (cilostamide and milrinone; Thomas et al., 2002; Sasseville et al., 2009; Albuz et al., 2010; Naruse et al., 2012) phosphodiesterase (PDE) type 3 inhibitors, or even stimulators of adenylate cyclase that potently increase whole COC and intra-oocyte cAMP levels (forskolin; Thomas et al., 2002). The combined use of cAMP modulators has also been proposed as an attempt to simulate physiological oocyte maturation (SPOM approach) that consists of a prematuration phase for $2 \mathrm{~h}$ in the presence of IBMX and forskolin (aiming to rapid increase cAMP levels in COCs), followed by an extended IVM culture $(30 \mathrm{~h})$ in the presence of cilostamide (Albuz et al., 2010). Another drug that efficiently induces meiotic block is butyrolactone I, which is a potent and specific inhibitor of cyclindependent kinases (cdks) but has few inhibitory effects on other protein kinases such as mitogen-activated protein (MAP) kinase (Kubelka et al., 2000). Although the blockade of meiosis in in vitro cultured oocytes has been extensively tested with the afore-mentioned drugs, little attention has been given to strategies such as inhibiting or delaying spontaneous nuclear maturation in vitro during oocyte transportation with the purpose of improving embryo production in a commercial OPU-IVP programme.

Thus, the aim of this study was to examine whether delaying precocious oocyte meiotic resumption with pharmacological (butyrolactone I, milrinone, or IBMX combined with forskolin) or biological (bFF) inhibitors during simulated transport of bovine oocytes for $6 \mathrm{~h}$ affects the oocyte acquisition of competence for embryonic development, as well as the quality of the embryos produced. Specifically, we assessed the following parameters: oocyte maturation rates; embryonic development to the blastocyst stage; total cell number in blastocysts; the frequencies of blastomeres undergoing apoptosis; and activity of caspases in the blastocysts.

\section{Materials and Methods}

\section{Chemicals and media}

Unless stated otherwise, chemicals were purchased from Sigma Chemical Co. (St. Louis, MO, USA). Plastic dishes and tubes were obtained from Corning Inc. (Acton, MA, USA). 
Both the medium for simulated transport of oocytes (which was called the IVM-like medium in the present study, as proposed by Ponderato et al., 2002) and the medium for IVM consisted of TCM199 (Gibco BRL, Grand Island, NY, USA) supplemented with $0.2 \mathrm{mM}$ sodium pyruvate, $25 \mathrm{mM}$ sodium bicarbonate, $50 \mu \mathrm{g} / \mathrm{ml}$ amikacin, $0.5 \mu \mathrm{g} / \mathrm{ml}$ follicular stimulating hormone ( $\mathrm{FSH}$; Follitropin-V, Bioniche Animal Health, Ontario, Canada), $100 \mathrm{IU} / \mathrm{ml}$ human chorionic gonadotropin (hCG; Vetecor, Hertape Calier, Juatuba, MG, Brazil). In addition, the medium for simulated transport was supplemented with $0.3 \%$ BSA because low concentrations of BSA during prematuration have been recommended in several studies in the literature (Adona \& Leal, 2004; Albuz et al., 2010), and then added with one type of meiosis inhibitor: $100 \mu \mathrm{M}$ butyrolactone I (Enzo Life Sciences, Inc., Farmingdale, NY, USA; Adona \& Leal, 2004); or $100 \mu \mathrm{M}$ forskolin + $500 \mu \mathrm{M}$ IBMX (Gibco BRL, Grand Island, NY, USA; Albuz et al., 2010); or $100 \mu \mathrm{M}$ milrinone (Naruse et al., 2012). Millimolar stock concentrations of the meiosis inhibitors (butyrolactone I, forskolin, IBMX, cilostamide and milrinone) were stored at $-20^{\circ} \mathrm{C}$ dissolved in anhydrous dimethyl sulphoxide (DMSO) solutions and diluted fresh for each experiment. The medium for IVM was supplemented with 10\% (v:v) FCS; Gibco BRL, Grand Island, NY, USA) or $0.6 \%$ BSA, depending on the experimental group. The IVF medium consisted of Tyrode's albumin lactate pyruvate (TALP) containing $0.2 \mathrm{mM}$ sodium pyruvate, $6 \mathrm{mg} / \mathrm{ml}$ fatty acid-free BSA, $25 \mathrm{mM}$ sodium bicarbonate, $13 \mathrm{mM}$ sodium lactate, $50 \mu \mathrm{g} / \mathrm{ml}$ amikacin, $4 \mu \mathrm{l} / \mathrm{ml}$ penicillamine-hypotaurine-epinephrine (PHE) solution (final concentrations of $20 \mu \mathrm{M}$ penicillamine, $10 \mu \mathrm{M}$ hypotaurine, and $2 \mu \mathrm{M}$ epinephrine), and $10 \mu \mathrm{g} / \mathrm{ml}$ heparin. The embryo in vitro culture (IVC) medium was synthetic oviductal fluid (SOF) supplemented with $0.2 \mathrm{mM}$ L-glutamine, $0.34 \mathrm{mM}$ sodium citrate, $2.8 \mathrm{mM}$ myo-inositol, 2\% MEM essential amino acid solution, 1\% MEM non-essential amino acid solution, $0.2 \mathrm{mM}$ sodium pyruvate, $50 \mu \mathrm{g} / \mathrm{ml}$ of amikacin, $5 \mathrm{mg} / \mathrm{ml}$ fraction $\mathrm{V}$ fatty acid-free BSA, and $2.5 \%$ FCS.

\section{Oocyte collection}

Abattoir-derived ovaries were transported to the laboratory in saline solution at $30-35^{\circ} \mathrm{C}$. Follicles measuring 3-8 $\mathrm{mm}$ in diameter were aspirated and the recovered COCs $(n=1385$, distributed among 10 replicates) were classified according to their morphological appearance. Bovine FF obtained from the pooled ovarian follicles was obtained at each routine and was centrifuged at $12,100 \mathrm{~g}$ for $5 \mathrm{~min}$ to remove cellular debris. During the selection period, the COCs were maintained in the centrifuged FF supplemented with $75 \mu \mathrm{g} / \mathrm{ml}$ penicillin/streptomycin to prevent the immediate resumption of meiosis. Oocytes with at least four layers of cumulus cells and a uniform cytoplasm were selected for the experiments. The interval between follicular aspiration and placing oocytes in culture was about $1 \mathrm{~h}$.

\section{Prematuration phase during simulated transport of oocytes}

Oocytes where the meiotic block was applied were cultured in vitro in transport medium with one of the following meiotic inhibitors: (1) BL group: $100 \mu \mathrm{M}$ butyrolactone I; (2) MR group: $100 \mu \mathrm{M}$ milrinone; or (3) mSPOM group: $100 \mu \mathrm{M}$ forskolin $+500 \mu \mathrm{M}$ IBMX (unlike the $2 \mathrm{~h}$ of prematuration proposed by Albuz et al. (2010) for the SPOM approach, the duration of the prematuration culture in the present study was $6 \mathrm{~h}$, so that we named this group as modified (m) SPOM). In the fourth experimental group (bFF group), oocytes were cultured in pure centrifuged bFF supplemented with $75 \mu \mathrm{g} / \mathrm{ml}$ penicillin/streptomycin. Oocytes were cultured in cryogenic vials (20-25 oocytes per vial) containing $100 \mu \mathrm{l}$ of transport medium or bFF recovered with mineral oil and gassed with a gaseous mixture of $5 \% \mathrm{O}_{2}, 5 \% \mathrm{CO}_{2}$ and $90 \% \mathrm{~N}_{2}$. The cryogenic vials were packed in a portable incubator (Thawing Unit MT $35 / 42$, Minitub) at $38.5^{\circ} \mathrm{C}$ during $6 \mathrm{~h}$. This period of time was defined based on the efficacy of IBMX, which was reported to be effective in maintaining meiosis blocked for up to $8 \mathrm{~h}$ (Barretto et al., 2007).

\section{In vitro maturation}

After simulation of transport, blocked oocytes from $\mathrm{BL}, \mathrm{MR}$ and bFF groups were washed from the transport medium aimed at complete removal of the meiotic inhibitors and cultured in $100-\mu l$ droplets of IVM medium supplemented with 0.6\% BSA (20-25 oocytes per droplet) under mineral oil for additional $18 \mathrm{~h}$ in a bench top $\mathrm{CO}_{2}$ incubator (Thermo Fisher Scientific, Marietta, Ohio, USA) at $38.5^{\circ} \mathrm{C}$, maximum humidity and atmosphere of $5 \% \mathrm{CO}_{2}$ in air (standard IVM). Oocytes from mSPOM group were washed from transport medium and cultured in 100- $\mu$ l droplets of IVM medium supplemented with $0.6 \%$ BSA and $20 \mu \mathrm{M}$ cilostamide (Enzo Life Sciences, Inc., Farmingdale, NY, USA), as proposed by Albuz et al. (2010) in the SPOM approach, under the same conditions used for the other groups.

There were three control groups in this study: (1) Control Lab FCS: oocytes were matured in 100- $\mu 1$ droplets of IVM medium supplemented with $10 \%$ FCS for $24 \mathrm{~h}$ in a standard IVM culture (in a $\mathrm{CO}_{2}$ incubator at $38.5^{\circ} \mathrm{C}$ under an atmosphere of $5 \% \mathrm{CO}_{2}$ in air with maximum humidity); (2) Control Lab BSA: oocytes were matured in 100- $\mu 1$ droplets of IVM medium 


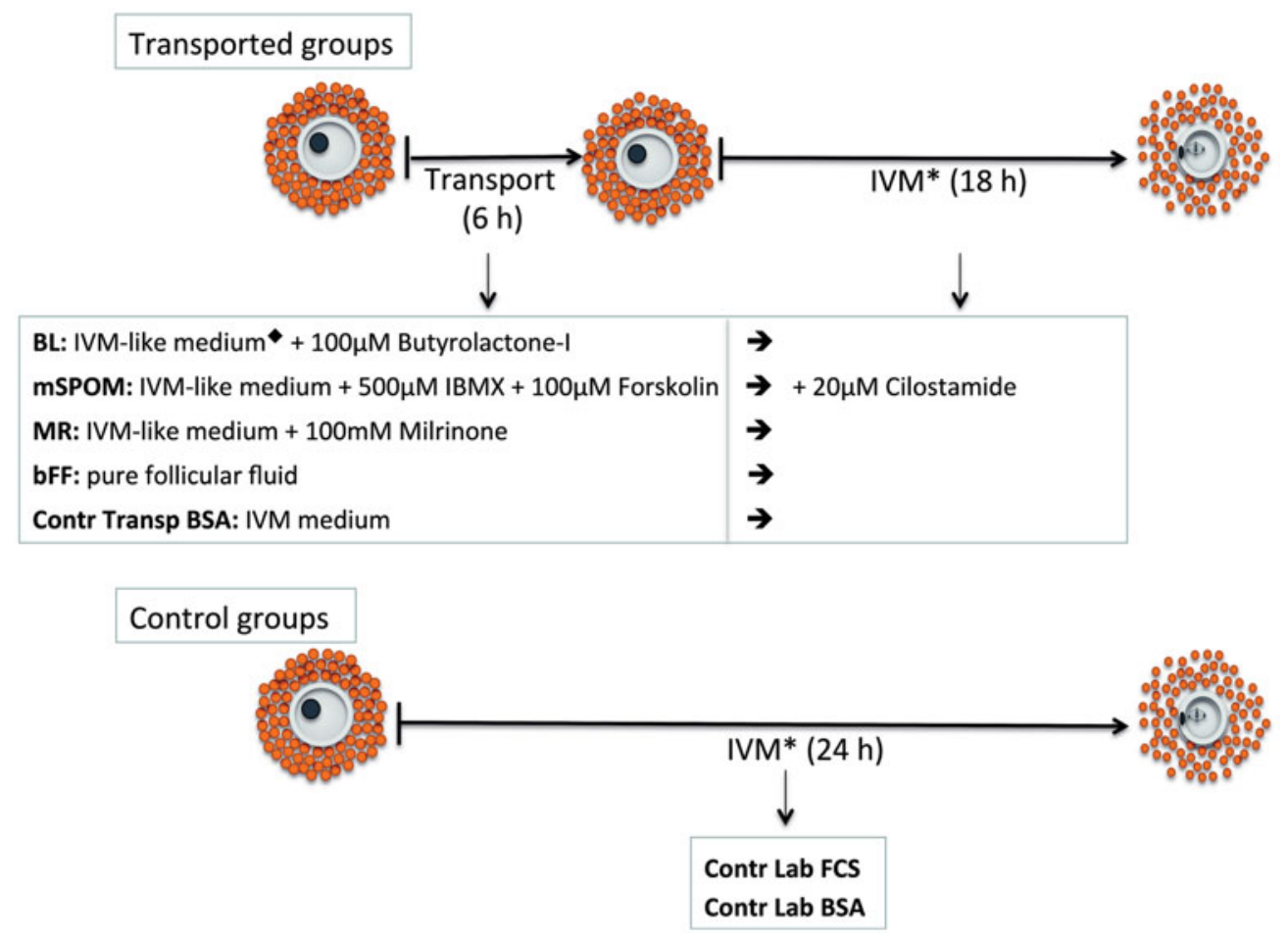

Figure 1 Schematic representation of the experimental design. ${ }^{*} \mathrm{IVM}$ was carried out in a $\mathrm{CO}_{2}$ incubator at $38.5^{\circ} \mathrm{C}$ under an atmosphere of $5 \% \mathrm{CO}_{2}$ in air with maximum humidity, in IVM medium (TCM199 with 0.2 mM sodium pyruvate, 25 mM sodium bicarbonate, $50 \mu \mathrm{g} / \mathrm{ml}$ amikacin, $0.5 \mu \mathrm{g} / \mathrm{ml} \mathrm{FSH}$, and $100 \mathrm{IU} / \mathrm{ml} \mathrm{hCG)} \mathrm{supplemented} \mathrm{with} 0.6 \%$ BSA in all treatments, with the exception of Contr FCS group which was supplemented with 10\% FCS. IVM-like medium: the same composition of IVM medium, supplemented with $0.3 \%$ bovine serum albumin (BSA).

supplemented with $0.6 \%$ BSA for $24 \mathrm{~h}$ in standard IVM; and (3) Control Transp BSA: oocytes were matured in cryogenic vials containing $100 \mu$ l of IVM medium supplemented with $0.6 \%$ BSA in the portable incubator (Minitub®) for $6 \mathrm{~h}$ (simulated transport), and then transferred to the $\mathrm{CO}_{2}$ incubator (standard IVM in 100$\mu \mathrm{l}$ droplets of the same IVM medium) for additional $18 \mathrm{~h}$ (total duration of culture $=24 \mathrm{~h}$ ).

The schematic representation of the experimental groups is shown in Fig. 1.

\section{Assessment of nuclear maturation}

At the end of the cultures, the frequencies of nuclear maturation were assessed as previously described in Rocha-Frigoni et al. (2016). Briefly, a sample of COCs was collected after follicle removal $(0 \mathrm{~h})$, after transportation $(6 \mathrm{~h})$, and after IVM ( $24 \mathrm{~h}$ for the control groups or $+18 \mathrm{~h}$ for the transported groups). After stripping from the cumulus cells and fixation in $4 \%(\mathrm{w} / \mathrm{v})$ paraformaldehyde solution for $10 \mathrm{~min}$ at room temperature, oocytes were stained with Hoechst $33342(1 \mu \mathrm{g} / \mathrm{ml})$ in the dark for $15 \mathrm{~min}$ at room temperature. Stained oocytes were evaluated under an epifluorescence inverted microscope (IX51; Olympus, Tokyo, Japan; $404 \mathrm{~nm}$ excitation and $526 \mathrm{~nm}$ emission wavelength) to determine meiosis stage, and those classified as germinal vesicle $(\mathrm{GV})$ were considered to be immature, while those classified as metaphase II (MII) were considered to be mature.

\section{In vitro fertilization and embryo culture}

Motile spermatozoa were obtained by centrifuging frozen-thawed semen on a Percoll (GE Healthcare, Bio-Science, Uppsala, Sweden) discontinuous density gradient $(250 \mu \mathrm{l}$ of $45 \%$ Percoll over $250 \mu \mathrm{l}$ of $90 \%$ Percoll in a $1.5-\mathrm{ml}$ microtube) for $7 \mathrm{~min}$ at $2500 \mathrm{~g}$ at room temperature. Sperm cells were added to the fertilization droplet at $2 \times 10^{6}$ cells $/ \mathrm{ml}$. The COCs (2025 per $100-\mu 1$ droplet covered with mineral oil) and the spermatozoa were coincubated for $18 \mathrm{~h}$ at $38.5^{\circ} \mathrm{C}$, maximum humidity and atmosphere of $5 \% \mathrm{CO}_{2}$ in air. The day of fertilization was defined as day 0 .

Following fertilization, the presumptive zygotes were partially stripped of excess cumulus cells and transferred to $100-\mu 1$ droplets of IVC medium covered with mineral oil, at $38.5^{\circ} \mathrm{C}$ under an atmosphere of $5 \% \mathrm{CO}_{2}$ in air, with maximum humidity. The cleavage rates were assessed under a stereomicroscope at $72 \mathrm{~h}$ post-insemination (h.p.i.; day 3 ), and the blastocyst development rates were recorded at 168 h.p.i. (day 7). 


\section{TUNEL (terminal deoxinucleotil transferase uracil nick end labelling) labelling}

The TUNEL assay was performed in blastocysts and early blastocysts ( $n=158$, distributed in 10 replicates) harvested at day 7, using the In Situ Cell Death Detection Kit (Fluorescein; Roche Diagnostics Corp., Indianapolis, IN, USA) according to manufacturer information and as previously described in Rocha-Frigoni et al. (2014). Labelling of TUNEL and Hoechst 33342 nuclei was observed using an inverted microscope (IX51; Olympus, Tokyo, Japan). Nuclei with greenyellow fluorescence at an excitation wavelength of 440$520 \mathrm{~nm}$ and an emission wavelength of 484-585 nm were considered TUNEL positive (fragmented DNA), whereas blue florescence at an excitation wavelength of 330-385 nm and an emission wavelength of $420 \mathrm{~nm}$ (Hoechst 33342) indicated the total number of cells.

\section{Detection of caspase-3 and caspase-7 activity}

Activity of caspases-3 and -7 was measured in expanded blastocysts $(n=175$, distributed in 10 replicates) harvested at day 7 using a fluorescent inhibitor of caspases (FLICA) by Image-iT LIVE Red Caspase-3 and -7 Detection Kit (Molecular Probes, Invitrogen, OR, USA), according to the manufacturer instructions. Briefly, the embryos were incubated in $10-\mu \mathrm{l}$ of FLICA working solution for $60 \mathrm{~min}$ at $37^{\circ} \mathrm{C}$ in a humid chamber and protected from light. After, the blastocysts were washed in a 1X buffer solution and fixed in $100-\mu \mathrm{l}$ drops of $4 \%(\mathrm{w} / \mathrm{v})$ paraformaldehyde solution for $40 \mathrm{~min}$ at room temperature. Subsequently, embryos were washed twice in PBS-PVP and mounted on $10 \%(\mathrm{w} / \mathrm{v})$ poly-L-lysine coated slides using $10-\mu 1$ drops of antifade, and coverslips were placed. The stained embryos were immediately visualized under an inverted microscope, with excitation and emission wavelengths of 515-575 nm and 560-680 nm, respectively. The recorded images were further analyzed running Q-Capture Pro imaging software (version 5.0.1.26, Media Cybernetics, Inc.) to quantify the fluorescence signal intensities (pixels). The means of the fluorescence intensity were plotted graphically with error bars representing the standard error of the mean (SEM).

\section{Statistical analysis}

Number of COCs and embryos per treatment and replicate numbers are provided in the tables and figures legends for each experiment. Treatment effects were assessed by analysis of variance (ANOVA) and the mean values were compared using Tukey's test, using JMP statistical software version 5.0.1a (SAS Inst. Inc., Cary, NC, USA). Data stated as percentages were arcsine-transformed prior to conducting an ANOVA. The level of statistical significance was set at $P<0.05$.

\section{Results}

After $6 \mathrm{~h}$ of simulated transport, the GV rates (Fig. 2A) of the oocytes from Contr Lab FCS $(20.5 \pm 3.0 \%)$ and Contr Transp BSA $(28.1 \pm 4.8 \%)$ groups were lower $(P<0.05)$ than that of the Contr Lab BSA $(51.6 \pm 4.3 \%)$, $\mathrm{BL}(55.0 \pm 5.3 \%)$ and $\mathrm{mSPOM}$ groups $(51.8 \pm 3.7 \%)$, but none of these rates differ $(P>0.05)$ from that of the oocytes transported in a medium supplemented with MR $(47.2 \pm 7.5 \%)$ and bFF (47.3 $\pm 4.7 \%)$. After $6 \mathrm{~h}$ of simulated transport followed by in vitro maturation for $18 \mathrm{~h}$ (Fig. 2B), most of the oocytes reached the MII stage and there were no differences between treatments $(P>0.05)$.

The effects of supplementation with meiotic blockers during simulated transport of oocytes on the rates of cleavage and development to the blastocyst stage are given in Table 1. Higher cleavage rates $(P<0.05)$ were obtained in Control Lab FCS group $(84.5 \pm 5.3 \%)$ compared with the other groups $(59.6 \pm 3.4 \%$ to $70.9 \pm 2.3 \%$ ). Embryonic development to the blastocyst stage was higher $(P<0.05)$ in the Control Lab FCS group $(39.8 \pm 4.7 \%)$ than in the Control Transp BSA $(22.7 \pm 3.4 \%)$ and MR $(21.6 \pm 2.3 \%)$ groups. However, all these groups were similar $(P>0.05)$ to Control Lab BSA $(28.8 \pm 2.7 \%)$, BL $(23.6 \pm 3.3 \%), \mathrm{mSPOM}$ $(24.1 \pm 3.2 \%)$ and bFF $(25.1 \pm 4.0 \%)$.

The total number of blastomeres in day-7 early blastocysts and blastocysts (Table 2) was higher $(P<0.05)$ in the Control Lab FCS group $(85.2 \pm 5.6)$ than in Control Lab BSA (53.6 \pm 2.9$)$, Control Transp BSA (55.5 \pm 4.4$)$, BL $(58.2 \pm 3.0), \operatorname{mSPOM}(57.9 \pm 4.9)$ and MR (59.2 \pm 3.9$)$, but all these treatments did not differ $(P>0.05)$ from bFF group $(67.7 \pm 4.2)$. However, there was no difference $(P>0.05)$ in the percentage of apoptotic cells (proportion of TUNEL-positive blastomeres) among the treatments (range, $12.3 \pm 2.0 \%$ to $15.7 \pm 1.7 \%$ ).

As shown in Fig. 3 , there were no effects $(P>0.05)$ of supplementation with meiotic blockers during simulated transport of oocytes in the activity of caspases-3 and -7 evaluated in day-7 expanded blastocysts (range, $139.0 \pm 3.2$ to $152.4 \pm 6.5$, expressed in fluorescence intensity of pixels).

\section{Discussion}

The results obtained in the present study indicated that the resumption of nuclear maturation was affected by the macromolecular supplementation, as 


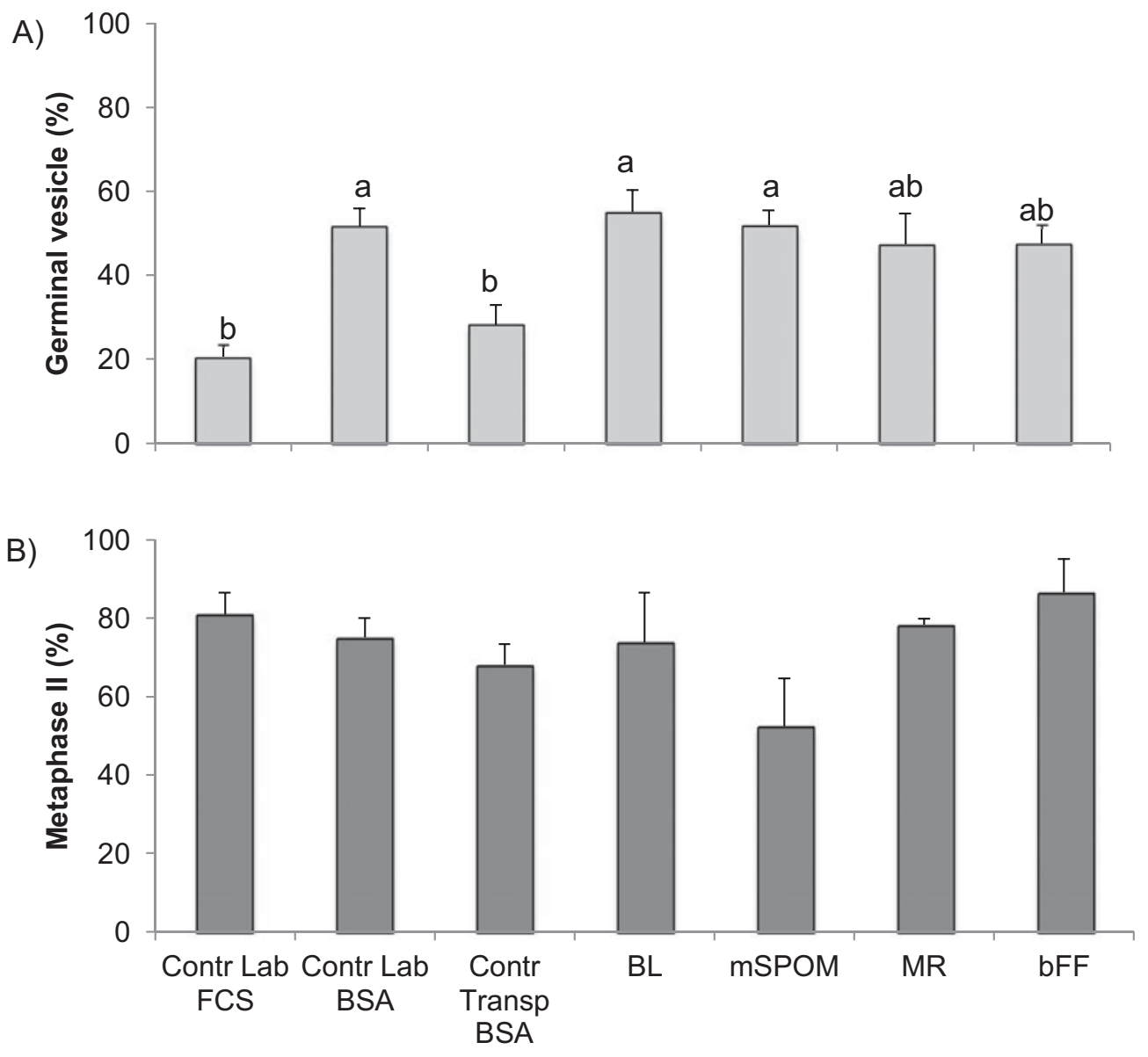

Figure 2 Meiotic progression of bovine oocytes cultured with different meiotic blockers during simulated transport. ( $A$ ) germinal vesicle rates after simulated transport for $6 \mathrm{~h}$; and $(B)$ metaphase II rates after simulated transport for $6 \mathrm{~h}$ and subsequently in vitro matured for additional $18 \mathrm{~h}$. In total, 20-30 oocytes were evaluated per group, distributed in at least three replicates. The data represent the means \pm standard error of the mean (SEM). Mean values followed by different letters differ significantly $(P<0.05)$ according to Tukey's test.

the replacement of FCS by BSA in the IVM medium promoted a delay in the resumption of meiosis in those oocytes cultured throughout the period within the incubator. A relationship between fatty acid $\beta$ oxidation and nuclear maturation was previously demonstrated (Paczkowski et al., 2013; Del Collado et al., 2015; Downs et al., 2009). According to Dunning et al. (2010), it is hypothesized that maturation in the presence of FCS results in higher oxidation rate when compared with IVM supplemented with BSA only, as a result of a possible lack of $\mathrm{L}$-carnitine from medium composed solely of BSA. Thus, as L-carnitine plays a crucial role in the $\beta$-oxidation pathway by catalyzing the transport of fatty acids to the mitochondrial matrix for the generation of ATP (Downs et al., 2009; Dunning et al., 2010), a possible decrease in oxidation could explain the delay in the resumption of meiosis observed in oocytes matured in the presence of BSA in the present study. However, despite the initial delay in resumption of meiotic maturation after replacement of the FCS by BSA, there was no difference among treatments in the rate of oocytes reaching MII stage (conclusion of meiosis) at the end of IVM cultures. Data from the literature are controversial, because although previous reports did not found impairment in the nuclear maturation and blastocyst development rates (Russell et al., 2006; Mingoti et al., 2011), other studies demonstrated that BSA slows nuclear maturation and reduces embryo production when compared with FCS (Del Collado et al., 2015; Ali et al., 2004). It is likely that different types of procedures and constituents of culture medium between different laboratories may mitigate or potentiate the effects of macromolecule substitution on the maturation and acquisition of competence in in vitro matured oocytes and, in the present study, the culture conditions appear to have been appropriate, regardless of the macromolecule used.

Besides the effects of macromolecular supplementation, the present results also demonstrated an effect 
Table 1 Cleavage rates and embryonic development to the blastocyst stage after fertilization of bovine oocytes treated with different meiotic blockers during $6 \mathrm{~h}$ of simulated transport

\begin{tabular}{llll}
\hline Treatments* & Oocytes $(n)$ & ${\text { Cleavage }(\%)^{\dagger}}^{\left.\text {Blastocysts }^{*} \%\right)^{\ddagger}}$ \\
\hline Control Lab FCS & 144 & $84.5 \pm 5.3^{\mathrm{a}}$ & $39.8 \pm 4.7^{\mathrm{a}}$ \\
Control Lab BSA & 239 & $69.5 \pm 2.9^{\mathrm{b}}$ & $28.8^{\mathrm{a}} \pm 2.7^{\mathrm{a}, \mathrm{b}}$ \\
Control Transp BSA & 233 & $70.9 \pm 2.3^{\mathrm{b}}$ & $22.7 \pm 3.4^{\mathrm{b}}$ \\
BL & 230 & $68.9 \pm 2.7^{\mathrm{b}}$ & $23.6 \pm 3.3^{\mathrm{a}, \mathrm{b}}$ \\
mSPOM & 231 & $65.8 \pm 2.7^{\mathrm{b}}$ & $24.1 \pm 3.2^{\mathrm{a}, \mathrm{b}}$ \\
MR & 233 & $64.5 \pm 2.6^{\mathrm{b}}$ & $21.6 \pm 2.3^{\mathrm{b}}$ \\
bFF & 243 & $59.6 \pm 3.4^{\mathrm{b}}$ & $25.1 \pm 4.0^{\mathrm{a}, \mathrm{b}}$ \\
\hline
\end{tabular}

${ }^{\dagger}$ Data are expressed as the mean \pm standard error of the mean (SEM) of 10 independent replicates. ${ }^{\ddagger}$ Mean values followed by different letters in the same column differ significantly $(P<0.05)$ according to Tukey's test. Cleavage $(72 \mathrm{hpi})$ and embryonic development to the blastocyst stage (168 hpi) of bovine embryos obtained after in vitro fertilization of oocytes matured according to the treatments*: Control Lab FCS: standard IVM in medium with 10\% FCS for 24 h; Control Lab BSA: standard IVM in medium with $0.6 \%$ BSA for 24 h; Control Transp BSA: simulated transport in medium with $0.6 \%$ BSA for 6 $\mathrm{h}$ followed by standard IVM in medium with $0.6 \%$ BSA for additional $18 \mathrm{~h}$; BL: simulated transport in medium containing $100 \mu \mathrm{M}$ butyrolactone I for $6 \mathrm{~h}$ followed by standard IVM in medium with $0.6 \%$ BSA for additional $18 \mathrm{~h}$; mSPOM: simulated transport in medium containing $100 \mu \mathrm{M}$ forskolin $+500 \mu \mathrm{M}$ IBMX for $6 \mathrm{~h}$ followed by standard IVM in medium with $0.6 \%$ BSA and $20 \mu \mathrm{M}$ cilostamide for additional $18 \mathrm{~h}$; MR: simulated transport in medium containing $100 \mu \mathrm{M}$ milrinone for $6 \mathrm{~h}$ followed by standard IVM in medium with $0.6 \%$ BSA for additional $18 \mathrm{~h}$; bFF: simulated transport in pure centrifuged bovine follicular fluid for $6 \mathrm{~h}$ followed by standard IVM in medium with $0.6 \%$ BSA for additional $18 \mathrm{~h}$.

Table 2 Total number of cells and apoptosis rate of day-7 early blastocysts and blastocysts obtained after fertilization of bovine oocytes treated with different meiotic blockers during $6 \mathrm{~h}$ of simulated transport

\begin{tabular}{llll}
\hline Treatments* & Embryos $(n)$ & Total number of cells & Apoptosis rate (\%) \\
\hline Control Lab FCS & 17 & $85.2 \pm 5.6^{\mathrm{a}}$ & $12.6 \pm 1.6$ \\
Control Lab BSA & 23 & $53.6 \pm 2.9^{\mathrm{b}}$ & $12.4 \pm 2.3$ \\
Control Transp BSA & 23 & $55.5 \pm 4.4^{\mathrm{b}}$ & $12.8 \pm 1.7$ \\
BL & 21 & $58.2 \pm 3.0^{\mathrm{b}}$ & $15.7 \pm 1.7$ \\
mSPOM & 19 & $57.9 \pm 4.9^{\mathrm{b}}$ & $12.3 \pm 2.0$ \\
MR & 26 & $59.2 \pm 3.9^{\mathrm{b}}$ & $14.2 \pm 1.6$ \\
bFF & 29 & $67.7 \pm 4.2^{\mathrm{a}, \mathrm{b}}$ & $14.1 \pm 2.3$ \\
\hline
\end{tabular}

Data are expressed as the mean \pm standard error of the mean (SEM) of 10 independent replicates. Mean values followed by different letters in the same column differ significantly $(P<0.05)$ according to Tukey's test. *Bovine early blastocysts and blastocysts obtained after in vitro fertilization of oocytes matured according to the treatments: Control Lab FCS: standard IVM in medium with 10\% FCS for $24 \mathrm{~h}$; Control Lab BSA: standard IVM in medium with 0.6\% BSA for 24 h; Control Transp BSA: simulated transport in medium with $0.6 \%$ BSA for $6 \mathrm{~h}$ followed by standard IVM in medium with $0.6 \%$ BSA for additional $18 \mathrm{~h}$; BL: simulated transport in medium containing $100 \mu \mathrm{M}$ Butyrolactone I for $6 \mathrm{~h}$ followed by standard IVM in medium with $0.6 \%$ BSA for additional $18 \mathrm{~h}$; mSPOM: simulated transport in medium containing $100 \mu \mathrm{M}$ forskolin + $500 \mu \mathrm{M}$ IBMX for $6 \mathrm{~h}$ followed by standard IVM in medium with $0.6 \%$ BSA and $20 \mu \mathrm{M}$ cilostamide for additional $18 \mathrm{~h}$; MR: simulated transport in medium containing $100 \mu \mathrm{M}$ milrinone for $6 \mathrm{~h}$ followed by standard IVM in medium with $0.6 \%$ BSA for additional $18 \mathrm{~h}$; bFF: simulated transport in pure centrifuged bovine follicular fluid for $6 \mathrm{~h}$ followed by standard IVM in medium with $0.6 \%$ BSA for additional $18 \mathrm{~h}$.

of culture conditions on the meiosis progression. Interestingly, it was observed that although supplementation of IVM medium with BSA had retarded the meiosis resumption of oocytes cultured throughout the period within the incubator (Contr Lab BSA group), it was accelerated when oocytes were cultured in the presence of BSA during $6 \mathrm{~h}$ of simulated transport (Contr Transp BSA group). According to Ambrogi et al. (2017), transportation of oocytes in incubators with an uncontrolled gaseous atmospheric tension can lead to alterations in the $\mathrm{pH}$ of the medium and increase the activity of oxidases, culminating in increased ROS production (Guérin et al., 2001; Guemra et al., 2014). Previous reports have demonstrated that $\mathrm{H}_{2} \mathrm{O}_{2}$ acts as a signalling molecule that modulates various aspects of oocyte physiology, including the blockage and resumption of meiosis (Combelles et al., 2009; Pandey et al., 2010). Thus, a possible increase in the intracellular $\mathrm{H}_{2} \mathrm{O}_{2}$ concentration would signal the resumption of meiosis, which could explain the acceleration of meiosis resumption observed in the transported oocytes. Conversely, no such acceleration 


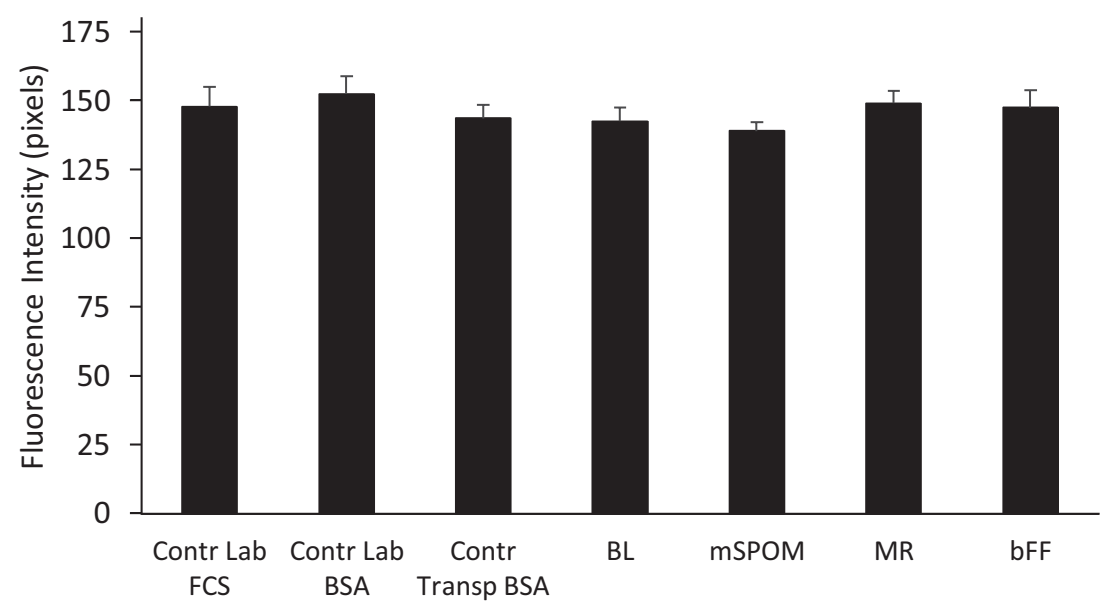

Figure 3 Caspase-3 and caspase-7 activity in bovine day-7 expanded blastocysts derived from oocytes cultured with different meiotic blockers during simulated transport for $6 \mathrm{~h}$ and subsequently in vitro matured for $18 \mathrm{~h}$. In total, 20-30 blastocysts were evaluated per group, distributed in 10 replicates. The data represent the means \pm standard error of the mean (SEM). There was no difference among treatments $(P>0.05$; Tukey's test).

was observed in the resumption of meiosis when oocytes were submitted to simulated transport for $6 \mathrm{~h}$ in IVM-like medium supplemented with BSA and butyrolactone I or forskolin + IBMX (BL and mSPOM groups, respectively), demonstrating the efficiency of these meiosis blockers to delay the resumption of meiosis, even in the presence of gonadotropins.

The meiosis block using butyrolactone I during the transport of oocytes was first suggested by Guemra et al. (2014) who demonstrated that this procedure is a promising alternative for the transport of oocytes during several hours (up to $19 \mathrm{~h}$ ), keeping their quality without interfering in embryo production and pregnancy rates. However, these authors have transported oocytes in a poor medium (TCM199 medium with $50 \mathrm{mg} / \mathrm{ml}$ gentamicin, $0.2 \mathrm{mM}$ pyruvate and $100 \mathrm{mM}$ cysteamine), and in the present approach we used an enriched prematuration medium identical to the IVM medium used routinely in our laboratory (Rocha-Frigoni et al., 2016) with the only difference of the addition of the inhibitors and the substitution of FCS for BSA in low concentration $(0.3 \%)$. The rationale behind this approach, that was previously proposed by Ponderato et al. (2002), is to use for prematuration a medium that is known to be well suited for oocyte culture with the objective to stimulate the process of capacitation during the inhibition period. In the present study, the output of embryos derived from oocytes that were transported in the absence of meiotic blockers (Control Transp BSA group) was lower than those matured throughout the period in the $\mathrm{CO}_{2}$ incubator in the presence of FCS; however, the inclusion of butyrolactone I in the transport medium partially improved the transport conditions, because blastocysts rates of BL group was similar to those of the
Control Lab FCS and Control Transp BSA groups. Thus, with this approach (transport of oocytes in IVM-like medium in the presence of butyrolactone I), we were able to partially improve the prematuration conditions because, although the improvement had not been very expressive, it allowed embryonic development to the blastocyst stage to be similar to the standard group of our laboratory (Contr Lab FCS).

Similarly, a partial improvement in the transport conditions was also observed when oocytes were transported in IVM-like medium supplemented with IBMX and forskolin and then matured in vitro in the presence of cilostamine (mSPOM group), because although blastocysts rates in this group did not differ from the Control Transp BSA group, they were also similar to the Control Lab FCS group. The addition of forskolin to the prematuration step of the SPOM approach is necessary to generate a rapid and large increase in cAMP that resembles the in vivo increase in COC cAMP levels and this treatment promotes long-acting and significant effects on subsequent COC functions and developmental programming of the oocyte, mainly due to a prolonging interaction between the oocyte and its surrounding cumulus cells in vitro (Albuz et al., 2010). Thus, although no substantial improving in the oocyte developmental outcomes were observed in the present study, results demonstrated that transporting oocytes in the prematuration condition of mSPOM approach during $6 \mathrm{~h}$ seems to be an interesting strategy for improving, at least partially, the developmental competence of the resulting mature oocyte.

As for cilostamide, milrinone is an oocyte-specific PDE type 3 inhibitor (PDE3) (Thomas et al., 2002; Sasseville et al., 2009; Naruse et al., 2012), however studies have demonstrated that cilostamide is more 
effective than milrinone in maintaining meiotic arrest in bovine oocytes (Tsafiri et al., 1996). In contrast with other inhibitors used in the present study, milrinone has not proven to be a good supplement for the transporting medium: although the output of embryos derived from this treatment was similar to Control Transp BSA and Control Lab BSA groups, it was lower than those matured throughout the entire period in the $\mathrm{CO}_{2}$ incubator in the presence of FCS. So, although it has not been detrimental, the lack of beneficial effects on the oocytes does not justify the use of this inhibitor during transport.

The effect of pure bFF on the developmental capacity of transported oocytes was also investigated in the present study. It is well known that FF contains factors that inhibit meiotic resumption, including the Müllerian inhibiting substance (Takahashi et al., 1986), linoleic acid (18:2) (Homa \& Brown, 1992) and hypoxanthine, a purine that maintains high levels of cAMP within the oocyte (Downs et al., 1989). More recently, evidence suggests that C-type natriuretic peptide (CNP) is likely to be the oocyte-meiosisinhibiting peptide present in the FF of a range of species, including bovine (Franciosi et al., 2014). Thus, the maintenance of oocytes in bFF during transport could be beneficial to prevent spontaneous resumption of meiosis, allowing more time to oocytes acquire competence for embryonic development during the period of inhibition. Similarly to results discussed above for $\mathrm{BL}$ and $\mathrm{mSPOM}$ groups, the transportation of oocytes during $6 \mathrm{~h}$ in pure bFF seems to be an interesting strategy for a partial increase in the developmental competence of the oocytes, as the embryonic development in this group was similar to those from Control Lab FCS group. However, despite the good results obtained with the bFF on the embryonic development, the results were very variable between each replicate in the present study (data not shown), which can be explained by its biological source. Probably the differences found in the composition of different samples of FF made maintenance of the results difficult, as occurs in different batches of FCS (Krisher et al., 1999).

Besides the evaluation of the competence of the oocytes for embryonic development until the blastocyst stage, in the present study we also evaluated the quality of the embryos. Apoptosis score was suggested as a good way to predict the viability of cultured embryos (Byrne et al., 1999; Pomar et al., 2005) because this phenomenon is important to remove damaged cells to allow the subsequent embryo development; however, high rates of apoptosis result in blockage of embryonic development (Paula-Lopes \& Hansen, 2002). As apoptosis in bovine IVP embryos is largely affected by culture conditions (Byrne et al., 1999), the frequencies of blastomeres undergoing apoptosis in embryos produced from oocytes transported for $6 \mathrm{~h}$ in the presence of meiotic blockers were evaluated in the present study and no differences regarding caspase3 and caspase-7 activity or nuclear fragmentation (TUNEL labelling) were found between treatments, suggesting that the conditions proposed for transport were satisfactory. The low frequencies of apoptosis in embryos in the present study may be explained by the presence of the proteins used as supplements of IVC medium (usually serum albumin from FCS or BSA), which participates in the antioxidant defence of embryos by trapping ROS and/or end-products of peroxidation due to its peroxyl-absorbing capacity (Guérin et al., 2001).

An interesting finding of the present study was the highest number of cells in embryos derived from oocytes matured in the presence of biological fluids, including the FCS during the entire period of IVM $(24 \mathrm{~h})$ and even the exposition to bFF during the short period of transport (only $6 \mathrm{~h}$ of exposure). The beneficial effect of bFF during IVM on cleavage rates and rates of blastocyst formation has been previously demonstrated (Romero-Arredondo \& Seidel, 1996; Ali et al., 2004) and the present study has demonstrated that also the quality of the embryos, as assessed by the number of cells, is improved by the undefined but apparently beneficial substances that are contained in the bFF. Thus, the present result suggests that protein supplementation during IVM can have profound effects on the quality of embryos, indicating that events that occurred during oocyte maturation were carried over to fertilization and early embryo development, as previously reported (Romero-Arredondo \& Seidel, 1996). However, while several molecules present in bFF, such as purines, growth factors, amino acids, steroids, and gonadotropins may be implicated as mediators of these benefits (Downs et al., 1989; Homa \& Brown, 1992; Romero-Arredondo \& Seidel, 1996; Lonergan et al., 1996; Bender et al., 2010; Franciosi et al., 2014), the active factors in bFF and their correspondent mechanisms of action remain to be determined.

In conclusion, this study tested the acquisition of embryonic developmental competence in bovine oocytes transported in a prematuration system with different types of inhibitors of meiosis resumption in IVM-like medium. The reason for this strategy is to promote the inhibition or delay of spontaneous nuclear maturation in vitro during oocyte transportation with the purpose of improving embryo production in a commercial OPU-IVP program. Supplementation of transport medium with BL or forskolin + IBMX (mSPOM approach) was efficient in delaying the resumption of meiosis during transport for $6 \mathrm{~h}$, as the percentage of GV oocytes from these treatments was higher than in those oocytes transported without meiosis blockers or cultured throughout the period within the incubator. 
The blockade of meiosis was reversible and most oocytes in all treatments reached the MII stage after $6 \mathrm{~h}$ of simulated transport followed by in vitro maturation for $18 \mathrm{~h}$. However, no improvement was observed in the acquisition of developmental competence of the oocytes, as well as the quality of the embryos produced was not affected. Although the approaches tested in this study did not promote significant differences in the quantity or quality of the resulting embryos, the results stimulate the development of new prematuration culture strategies during long-term oocyte transport (i.e. herds distant from the laboratory) with the aim of taking advantage of this time to improve their competence.

\section{Acknowledgements}

The present study was supported by São Paulo Research Foundation (FAPESP), Brazil. P.C.D.A. was supported by a scholarship from Coordenação de Aperfeiçoamento de Pessoal de Nível Superior (Capes), Brazil and G.Z.M. was awarded with scholarship from Conselho Nacional de Desenvolvimento Científico e Tecnológico (CNPq, \#306746/2012-3), Brazil. The authors thank Brasfrigo for donating the biological material used in the present study, and Adão A. Custodio and Alexandre J. Teixeira for technical support.

\section{References}

Adona, P.R. \& Leal, C.L.V. (2004). Meiotic inhibition with different cyclin-dependent kinase inhibitors in bovine oocytes and its effects on maturation and embryo development. Zygote 12, 197-204.

Albuz, F.K., Sasseville, M., Lane, M., Armstrong, D.T., Thompson, J.G. \& Gilchrist, R.B. (2010). Simulated physiological oocyte maturation (SPOM): a novel in vitro maturation system that substantially improves embryo yield and pregnancy outcomes. Hum. Reprod. 25, 2999-3011.

Ali, A., Coenen, K., Bousquet, D. \& Sirard, M.A. (2004). Origin of bovine follicular fluid and its effect during in vitro maturation on the developmental competence of bovine oocytes. Theriogenology 62, 1596-606.

Ambrogi, M., Dall'Acqua, P.C., Rocha-Frigoni, N.A.S., Leão, B.C.S. \& Mingoti, G.Z. (2017). Transporting bovine oocytes in a medium supplemented with different macromolecules and antioxidants: effects on nuclear and cytoplasmic maturation and embryonic development in vitro. Reprod. Dom. Anim. 52, 409-21.

Barceló-Fimbres, M., Campos-Chillón, L.F., Mtango, N.R., Altermatt, J., Bonilla, L., Koppang, R. \& Verstegen, J.P. (2015). Improving in vitro maturation and pregnancy outcome in cattle using a novel oocyte shipping and maturation system not requiring a $\mathrm{CO}_{2}$ gas phase. Theriogenology 84, 109-17.
Barretto, L.S.S., Caiado Castro, V.S.D., Garcia, J.M. \& Mingoti, G.Z. (2007). Role of roscovitine and IBMX on kinetics of nuclear and cytoplasmic maturation of bovine oocytes in vitro. Anim. Reprod. Sci. 99, 202-7.

Bender, K., Walsh, S., Evans, A.C.O., Fair, T. \& Brennan, L. (2010). Metabolite concentrations in follicular fluid may explain differences in fertility between heifers and lactating cows. Reproduction 139, 1047-55.

Bilodeau-Goeseels, S. (2012). Bovine oocyte meiotic inhibition before in vitro maturation and its value to in vitro embryo production: Does it improve developmental competence? Reprod. Dom. Anim. 47, 687-93.

Byrne, A.T., Southgate, J., Brison, D.R. \& Leese, H.J. (1999). Analysis of apoptosis in the preimplantation bovine embryos using TUNEL. J. Reprod. Fertil. 117, 97-105.

Combelles, M.H.C., Gupta, S. \& Agarwal, A. (2009). Could oxidative stress influence the in-vitro maturation of oocytes? Reprod. BioMed. Online 18, 864-80.

Del Collado, M., Saraiva, NZ., Lopes, F.L., Gaspar, R.C., Padilha, L.C., Costa, R.R., Rossi, G.F., Vantini, R. \& Garcia, J.M. (2015). Influence of bovine serum albumin and fetal bovine serum supplementation during in vitro maturation on lipid and mitochondrial behaviour in oocytes and lipid accumulation in bovine embryos. Reprod. Fertil. Dev. 28, 1721-32.

Downs, S.M., Daniel, S.A.J., Bomslaeger, E.A., Hoppe, P.C. \& Eppig, J.J. (1989). Maintenance of meiotic arrest in mouse oocytes by purines: Modulation of cAMP levels and cAMP phosphodiesterase activity. Gamete Res. 23, 323-34.

Downs, S.M., Mosey, J.L. \& Klinger, J. (2009). Fatty acid oxidation and meiotic resumption in mouse oocytes. Mol. Reprod. Dev. 76, 844-53.

Ducolomb, Y., González-Márquez, H., Fierro, R., Jiménez, I., Casas, E., Flores, D., Bonilla, E., Salazar, Z. \& Betancourt, M. (2013). Effect of porcine follicular fluid proteins and peptides on oocyte maturation and their subsequent effect on in vitro fertilization. Theriogenology 79, 896904.

Dunning, K.R., Cashman, K., Russell, D.L., Thompson, J.G., Norman, R.J. \& Robker, R.L. (2010). $\beta$-Oxidation is essential for mouse oocyte developmental competence and early embryo development. Biol. Reprod. 83, 909-18.

Franciosi, F., Coticchio, G., Lodde, V., Tessaro, I., Modina, S.C., Fadini, R., Dal Canto, M., Renzini, M.M., Albertini, D.F. \& Luciano, A.M. (2014). Natriuretic peptide precursor $C$ delays meiotic resumption and sustains gap junctionmediated communication in bovine cumulus-enclosed oocytes. Biol. Reprod. 91, 61.

Gilchrist, R.B. (2011). Recent insights into oocyte-follicle cell interactions provide opportunities for the development of new approaches to in vitro maturation. Reprod. Fertil. Dev. 23, 23-31.

Gilchrist, R.B. \& Thompson, J.G. (2007). Oocyte maturation: Emerging concepts and technologies to improve developmental potential in vitro. Theriogenology 67, 6-15.

Guemra, S., Santos, ES., Zanin, R., Monzani, P.S., Sovernigo, T.C., Ohashi, O.M., Leal, C.L.V. \& Adona, P.R. (2014). Effect of temporary meiosis block during prematuration of bovine cumulus-oocyte complexes on pregnancy rates in a commercial setting for in vitro embryo production. Theriogenology 81, 982-7. 
Guérin, P., Mouatassim, S.E.L. \& Ménézo, Y. (2001). Oxidative stress and protection against reactive oxygen species in the pre-implantation embryo and its surroundings. Hum. Reprod. Update 7, 175-89.

Hashimoto, S., Minami, N., Takakura, R. \& Imai, H. (2002). Bovine immature oocytes acquire developmental competence during meiotic arrest in vitro. Biol. Reprod. 66, 1696-701.

Homa, S.T. \& Brown, C.A. (1992). Changes in linoleic acid during follicular development and inhibition of spontaneous breakdown of germinal vesicles in cumulusfree bovine oocytes. J. Reprod. Fertil. 94, 153-60.

Krisher, R.L., Lane, M. \& Bavister, B.D. (1999). Developmental competence and metabolism of bovine embryos cultured in semi-defined and defined culture media. Biol. Reprod. 60, 1345-52.

Kubelka, M., Motlik, J., Schultz, R.M. \& Pavlok, A. (2000). Butyrolactone I reversibly inhibits meiotic maturation of bovine oocyte, without influencing chromosome condensation activity. Biol. Reprod. 62, 292-302.

Lonergan, P., Carolan, C., Van Langendonckt, A., Donnay, I., Khatir, H. \& Mermillod, P. (1996). Role of epidermal growth factor in bovine oocyte maturation and preimplantation embryo development in vitro. Biol. Reprod. 54, 1420-9.

Mingoti, G.Z., Castro, V.S.D.C., Méo, S.C., Barretto, L.S.S. \& Garcia, J.M. (2011). The effects of macromolecular and serum supplements and oxygen tension during bovine in vitro procedures on kinetics of oocyte maturation and embryo development. In Vitro Cell Dev. Biol. Anim. 47, 361-7.

Naruse, K., Iga, K., Shimizu, M., Takenouchi, N., Akagi, S., Somfai, T. \& Hirao, Y. (2012). Milrinone treatment of bovine oocytes during in vitro maturation benefits production of nuclear transfer embryos by improving enucleation rate and developmental competence. J. Reprod. Dev. 58, 476-83.

Paczkowski, M., Silva, E., Schoolcraft, W.B. \& Krisher, R.L. (2013). Comparative importance of fatty-acid $\beta$-oxidation to nuclear maturation, gene expression and glucose metabolism in mouse, bovine and porcine cumulus-oocyte complexes. Biol. Reprod. 88, 111.

Pandey, A.N., Tripathi, A., Premkumar, K.V., Shrivastav, T.G. \& Chaube, S.K. (2010). Reactive oxygen and nitrogen species during meiotic resumption from diplotene arrest in mammalian oocytes. J. Cell. Biochem. 111, 521-8.

Paula-Lopes, F.F. \& Hansen, P.J. (2002). Apoptosis is an adaptive response in bovine preimplantation embryos that facilitates survival after heat shock. Biochem. Biophys. Res. Commun. 295, 37-42.

Perry, G. (2015). 2013 Statistics of embryo collection and transfer in domestic farm animals. Embryo Transfer Newslett. 33, 14-26.
Pomar, F.J.R., Teerds, K.J., Kidson, A., Colenbrander, B., Tharasanit, T., Aguilar, B. \& Roelen, B.A.J. (2005). Differences in the incidence of apoptosis between in vivo and in vitro produced blastocysts of farm animal species: a comparative study. Theriogenology 63, 2254-68.

Ponderato, N., Crotti, G., Turini, P., Duchi, R., Galli, C. \& Lazzari, G. (2002). Embryonic and foetal development of bovine oocytes treated with a combination of butyrolactone I and roscovitine in an enriched medium prior to IVM and IVF. Mol. Reprod. Dev. 62, 513-8.

Rocha-Frigoni, N.A.S., Leão, B.C.S., Dall'Acqua, P.C. \& Mingoti, G.Z. (2016). Improving the cytoplasmic maturation of bovine oocytes matured in vitro with intracellular and/or extracellular antioxidants is not associated with increased rates of embryo development. Theriogenology 86, 1897-905.

Rocha-Frigoni, N.A., Leão, B.C., Nogueira, É., Accorsi, M.F. \& Mingoti, G.Z. (2014). Reduced levels of intracellular reactive oxygen species and apoptotic status are not correlated with increases in cryotolerance of bovine embryos produced in vitro in the presence of antioxidants. Reprod. Fertil. Dev. 26, 797-805.

Romero-Arredondo, A. \& Seidel, Jr, GE. (1996). Effects of follicular fluid during in vitro maturation of bovine oocytes on in vitro fertilization and early embryonic development. Biol. Reprod. 55, 1012-6.

Russell, D.F., Baqir, S., Bordignon, J. \& Betts, D.H. (2006). The impact of oocyte maturation media on early bovine embryonic development. Mol. Reprod. Dev. 73, 1255-70.

Sasseville, M., Albuz, FK., Côté, N., Guillemette, C., Gilchrist, R.B. \& Richard, F.J. (2009). Characterization of novel phosphodiesterases in the bovine ovarian follicle. Biol. Reprod. 81, 415-25.

Sirard, M.A., Richard, F. \& Mayes, M. (1998). Controlling meiotic resumption in bovine oocytes: a review. Theriogenology 49, 483-97.

Takahashi, M., Koide, S.S. \& Donahoe, PK. (1986). Müllerian inhibiting substance as oocyte meiosis inhibitor. Mol. Cell. Endo. 47, 225-34.

Thomas, R.E., Armstrong, D.T. \& Gilchrist, R.B. (2002). Differential effects of specific phosphodiesterase isoenzyme inhibitors on bovine oocyte meiotic maturation. Dev. Biol. 244, 215-25.

Tsafiri, A., Chun, SY., Zhang, R., Hsueh, A.J.W. \& Conti, M. (1996). Oocyte maturation involves compartmentalization and opposing changes of cAMP levels in follicular somatic and germ cells: studies using selective phosphodiesterase inhibitors. Dev. Biol. 178, 393-402.

van den Hurk, R. \& Zhao, J. (2005). Formation of mammalian oocytes and their growth, differentiation and maturation within ovarian follicles, Theriogenology $63,1717-51$. 